\title{
Role of ASOCT in Preoperative Assessment of Posterior Polar Cataract
}

\author{
Abhinav Dhami*, Nimratabajaj Dhami and Gobinder Singh Dhami \\ Retina and Cataract Services, Dhami Eye Hospital, India \\ *Corresponding Author: Abhinav Dhami, Retina and Cataract Services, Dhami Eye \\ Hospital, India.
}

Received: October 09, 2020

Published: October 30, 2020

(C) All rights are reserved by Abhinav Dhami., et al.

\begin{abstract}
Objective: Preoperative anterior segment optical coherence tomography in assessing posterior polar capsule integrity.

Method: A prospective observational study of 40 eyes of 25 patients. Patients with posterior polar cataracts undergoing elective cataract surgery were planned for ASOCT [Copernicus Revo (OCT NX700 Optopol technology) and the IOL master 700 (Carl Zeiss Meditec AG, Jena, Germany)] for determining the posterior capsular integrity and planned for phacoemulsification, by two surgeons during the period of January 2019 to December 2019. Posterior polar cataracts were categorized: Type 1- "continuous"; Type 2: abrupt, Type 3: "dehiscent".

Results: A total of 40 eyes (25 patients) were included. 30 eyes (75\%) were bilateral (15 patients) and 10 eyes (25\%) were unilateral patients. ASOCT findings noted were, type-1 PPC were observed to be 85\% (34 eyes) and 70\% (30 eyes) equally detected with both the IOL master 700 and the Optopol ASOCT, Type-2 PPC were $12.5 \%$ (5) and 15\% (6) of the cases and type-3 PPC were 2.5\% (1) with the IOL master 700 and 10\% (4) with the Optopol Revo ASOCT. The sensitivity and specificity for the IOL master 700 for predicting the PPC integrity was $90.32 \%$ and $66.27 \%$ each, and for the Optopol REVO ASOCT was $96.77 \%$ and $90 \%$ each. Intraoperatively, posterior capsular rent was noted in type-3 PPC, 2 cases (5\%).

Conclusion: AS-OCT provides a relevant insight for predicting preoperative PC deficits thus enabling better surgical planning and in anticipating PCR.
\end{abstract}

Keywords: Posterior Polar Cataracts (PPCs); Posterior Capsule (PC); Optical Coherence Tomography

\section{Introduction}

Posterior polar cataracts (PPCs) have an abnormal adhesion of the posterior capsule leading to a polar opacity with or without a preexisting weakness of the posterior capsule (PC) [1]. It is a form of congenital cataract with an prevalence of 3 to 5 in $1000[1,2]$. The importance of PPC is because it has a known risk for developing posterior capsular tear intraoperatively [2]. PPC presents as a central ring shaped plaque in the PC of the lens (concentric rings around the central opacity) (Figure 1) [2]. PPC consists of dysplastic lens fibers, thus forming the discoid PP plaque-like cataract [3]. Literature states a presence of PC underneath the PPC is doubtful, with the presence of an intact PC or a pre-existing PC deficit with abnormal adherence of the acellular opacity to the capsule. In $20 \%$ of the cases, a congenital deficit in the PC can be observed [4].

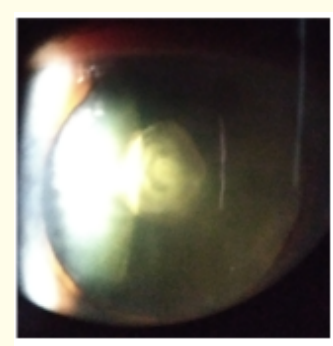

Figure 1: Slit lamp photograph showing the circular plaque in the central posterior part of the lens, depicting PPC. 
Even with newer techniques and better fluidic maintenance in cataract surgery, operating on PPC poses a challenge, as the reported incidence of posterior capsule rupture (PCR) in PPC ranges from $7.1 \%$ to $36 \%$ [5]. PCR can be noted intraoperatively during the various steps hydro-dissection, central nuclear emulsification, or spontaneously with sudden alterations in intraocular pressure (IOP) during insertion or removal of phaco hand piece [1]. As slit-lamp evaluations cannot accurately detect a preexisting PC dehiscence in PPC, therefore it's an apt knowledge on the integrity of PC preoperatively can provide an insight for improving surgical outcomes.

Diagnostic modalities, including preoperative anterior segment optical coherence tomography (AS-OCT), high-frequency ultrasound, and Scheimpflug imaging, have been used previously in literature to detect preexisting defects in the posterior capsule [6-8].

Herein we compare the efficacy of two different AS-OCT machines in predicting the posterior capsule integrity in PPC's, using the SOCT Copernicus Revo (OCT NX700 Optopol technology) and the IOL master 700 (Carl Zeiss Meditec AG, Jena, Germany) for planning optimal surgical outcomes.

\section{Materials and Methods}

The study was in accordance with the Declaration of Helsinki with an approval from the institutional review board. Informed consent were taken from all study participants. The study comprised patients visiting the outpatient department of Private clinic in Northern India, who were diagnosed with PPC and aspired for cataract surgery between January to December 2019.

Participants included in the study aged between 25 - 70 years. Patients had PPC on presentation with or without an associated nuclear sclerosis which were graded as per the Lens Opacities Classification System (LOCS). It was noted that PPC was the primary cause of visual impairment of 20/40 or less on the Snellen's chart. The exclusion criteria included, a differentiation from posterior subcapsular cataract, previous history of ocular trauma, or any preexisting corneal or retinal pathology.

A preoperative evaluation, including distance Snellen visual acuity assessment, IOP was measured with a Non-contact tonometer, and detailed slit-lamp evaluation for anterior and posterior segment was performed after pupillary dilation using 90-diopter lens.

AS-OCT imaging was conducted after a pupillary dilation of more than $6 \mathrm{~mm}$ and a scan length of $10.0 \mathrm{~mm}$ and a depth of 3.0 mm was obtained. The main focus was acquisition of both horizontal and vertical axial scans of the posterior one third of the lens for evaluating the PC integrity. For each eye, two investigating authors (A.D, G.S.D) used AS-OCT images to grade the preoperative integrity of the PC as type1-"continuous"; if the capsular margin was seen below the PPC (Figure 2a and 3a), Type 2- abrupt; if the capsular margin could not be seen just under the PPC but is intact beyond it (Figure $2 \mathrm{~b}$ and $3 \mathrm{~b}$ ), and Type 3- "dehiscent"; if the capsular margin is absent under the PPC with a defect or if it is discontinuous with protrusion of content in patellar fossa (Figure 2c and 3c).

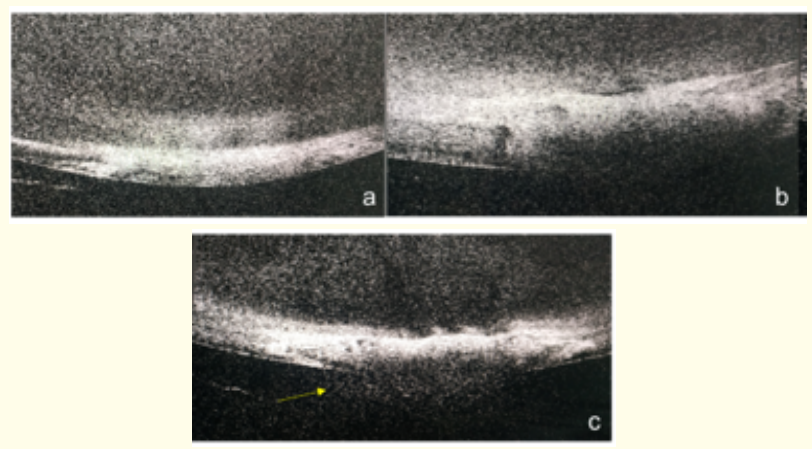

Figure 2: ASOCT scan of the Optopol Revo Nx 700 scan; a) showing type 1 PPC - "continuous"; with the capsular margin traced below the posterior polar cataract without any defect or discontinuity, b) ASOCT scan showing Type 2- abrupt; if the capsular margin could not be traced just under the posterior polar cataract but is seen beyond it, c) shows Type 3- "dehiscent"; (yellow arrow) the capsular margin could not be traced under the posterior polar cataract with a defect and protrusion of contents in patellar fossa.

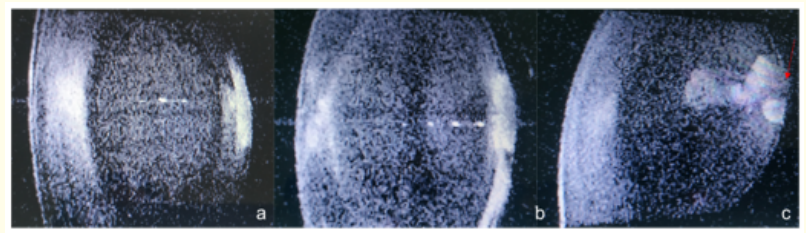

Figure 3: ASOCT scan of the IOL master 700 (Carl Zeiss Meditec AG, Jena, Germany) a) shows type 1 PPC-“continuous"; b) shows

Type 2- abrupt PPC wherein the posterior capsule cannot be traced under the PPC, c) shows type 3 PPC showing a bulge (red arrow) of PPC with central defect of the capsule.

IOL master 700 obtains a swept source OCT scan to measures axial length (AL), central corneal thickness (CCT), anterior cham- 
ber depth (ACD) and lens thickness (LT). The operator can evaluate the scan images and analyse the eye geometry, anterior and posterior capsule and central foveal architecture. Whereas Optopol Revo $\mathrm{Nx} 700$ machine uses ray tracing in the anterior segment 3D scan and calculates the refractive power of the cornea and the LT formula. It analyses parallel light beams being refracted according to the relevant refractive indices $(1.0,1.376,1.336)$, using the exact location of the refraction and the slope of the ocular surfaces. The location of the refraction is the main factor of the measuring surface slope, as both the anterior and posterior surfaces have a slight different principal planes, which in turn provides the image scan for the PC surface.

All eyes underwent phacoemulsification performed by experienced surgeons (A.D and G.S.D) and were performed with a topical anesthetic instillation (proparacaine eye drops (0.5\%) 5 minutes prior to surgery. Topical Moxifloxacin $0.3 \%$ eye drops were instilled every 2 hourly preoperatively in the eye to be operated. Care was taken to maintain complete asepsis, with preoperative instillation of povidone iodine $5 \%$ antiseptic in the conjunctival sac. with A temporal corneal sitting was used for performing all cataract surgeries. At first side-port incision was made followed with a selfsealing $2.8 \mathrm{~mm}$ clear corneal incision. The capsulorhexis was done using Utrata forceps with a rhexis size aimed between $5.5 \mathrm{~mm}-6.0$ $\mathrm{mm}$. Care was taken to avoid hydrodissection in all cases and hydrodelineation was performed to separate the central nuclear cataract from the peripheral cortex. The nuclear fragment was emulsified using a direct chop technique within the epi-nuclear cushion. During an exchange of instrument anterior chamber was instilled with ocular viscoelastic devices. The remaining peripheral cortex was removed using coaxial J-shaped irrigation/aspiration probe. The PPC plaque was removed with the irrigation/aspiration probe, care was taken to keep infusion flow low to prevent PCR during irrigation and aspiration. The anterior chamber stability was maintained throughout the surgery. All patients were planned for a 1-piece foldable intraocular lens (IOL) implantation with an intact capsular bag. In case of an intraoperative PCR, anterior vitrectomy with a cutter probe was done to clear the central pupil margins of vitreous and a 3-piece foldable IOL was implanted in the sulcus.

\section{Statistical analysis}

Descriptive statistics were analyzed for continuous variables and frequency distribution was interpreted for qualitative variables. Chi Square test for independence was used to calculate the p-value. All statistical analysis in the study were performed using SPSS software (version 14.0, SPSS, Inc). A P value of less than 0.05 was considered statistically significant.

\section{Results}

The total number of 40 eyes of 25 patients were studied. 30 eyes (75\%) were bilateral (15 patients) and 10 eyes (25\%) were unilateral patients. The number of eyes with nuclear sclerosis 1 were 24 (60\%), type 2: 10 (25\%), type 3 were 6 (15\%). The table 1 shows the patients demographic.

\begin{tabular}{|l|c|}
\hline Parameter & Value \\
\hline Mean Age of patients & $48.61 \pm 12.23$ years \\
\hline Age (Range) & $25-70$ years \\
\hline Males & $9(45 \%)$ \\
\hline Females & $11(55 \%)$ \\
\hline Bilateral eyes with PPC & $30(75 \%)$ \\
\hline Unilateral & $10(25 \%)$ \\
\hline In the bag IOL placement & $38(95 \%)$ \\
\hline Sulcus placement & $2(5 \%)$ \\
\hline Intraoperative intact capsule & $38(95 \%)$ \\
\hline $\begin{array}{l}\text { Posterior capsule dehiscence noted } \\
\text { intraoperatively }\end{array}$ & $2(5 \%)$ \\
\hline
\end{tabular}

Table 1: Demography of patients.

The preoperative ASOCT findings noted were, type-1 PPC were observed to be $85 \%$ (34 eyes) and 70\% (30eyes) with the IOL master 700 and the Optopol ASOCT, Type-2 PPC were $12.5 \%$ an $15 \%$ each and type-3 PPC were $2.5 \%$ with the IOL master 700 and 10 $\%$ with the Optopol Revo ASOCT. 2 cases of type-2 PPC as noted on IOL master 700 were confirmed to have a deficient capsule of Optopol Revo with no occurrence of intraoperative PCR. The p-value of less than $0.05 \%$ was observed in detecting the Types of PPC with p-value of 0.0002 (Ziess IOL master 700) and 0.00032 (Optopol Revo), denoting a significant correlation (Table 2).

\begin{tabular}{|l|c|c|c|c|}
\hline & Type 1 & Type 2 & Type 3 & P value \\
\hline $\begin{array}{l}\text { Ziess IOL master } \\
700(\mathrm{n}=40)\end{array}$ & $34(85 \%)$ & $5(12.5 \%)$ & $1(2.5 \%)$ & 0.0002 \\
\hline Optopol $(\mathrm{n}=40)$ & $30(70 \%)$ & $6(15 \%)$ & $4(10 \%)$ & 0.00032 \\
\hline PCR & 0 & 0 & $2(5 \%)$ & \\
\hline
\end{tabular}

Table 2: Demography and comparison of types of posterior polar on ASOCT machines. 
Intraoperatively no PCR was noted in Type 1 and type 2 PPC, while in type-3 PPC, 2 cases (5\%) had a PCR during Irrigation and aspiration for cortical matter removal and were managed with anterior vitrectomy and 3-piece intraocular lens placement in the sulcus with optic capture.

The sensitivity and specificity for the IOL master 700 for predicting the PPC integrity was $90.32 \%$ and $66.27 \%$ each, and for the Optopol REVO ASOCT was $96.77 \%$ and $90 \%$ each.

\section{Discussion and Conclusion}

The management of PPC is at times a difficult task even in the hands of most experienced anterior segment surgeons as $[1,2]$ the incidence of intraoperative PC dehiscence have been noted to be around 36\% [3-6]. Various means to identify a reliable method for PPC cases have been studied in literature with different machines, so that eyes at high risk versus low risk for PCR could be identified preoperatively but a definite method is still lacking in literature with a $100 \%$ specificity [5-7].

The reported incidence of the PCR rate was noted in literature is in the range of $7.1 \%$ to $36 \%$ [3-6], but in our series we observed a relatively lower incidence for intraoperative PCR (5\%).

In our study, our aim was to facilitate the use of AS-OCT on two different machines to analyze eyes with PPC and localize any PC deficits preoperatively. We observed $70-85 \%$ of eyes had intact PC (type-1), 12.5 - 15\% with no clear distinction of intact PC (type-2) and $2.5-10 \%$ with deficit PC (type-3) with bulge of the cortical content in the patellar fossa. In comparison to results described by Pavan G., et al. [6] wherein $87.5 \%$ had an intact PC and 8 eyes $(12.5 \%)$ showed dehiscent PC. In their series [6], they described two eyes where they were unable to trace the PC integrity because of the presence of multiple small points of PC deficits and labelled them as dehiscent PC, while in our study we have described them as type 2 PPC and observed no predisposition of such cases for developing PCR intraoperatively and it may be just be due to thickness of PPC wherein the ASOCT machine is unable to trace the PC.

Sachdev., et al. [8] used the ASOCT imaging of the CATALYS femtosecond laser system to determine the PPC integrity and observed, an intact capsule in $86 \%$ (43 eyes) cases and a dehiscent capsule in $14 \%$ (7 eyes) which similar in comparison with ASOCT with both devices in our study.
The striking finding in our study, which is in comparison with other studies in literature is the prevalence of negative predictive value of $100 \%$, implicating a minimal chance of developing intraoperative PCR in the presence of an intact PC on AS-OCT preoperatively. Even in Type 2 PPC, we report a 100\% negative predictive value. This information acts as a guide, allowing the operating surgeon to have a better-informed preoperative counselling with the patient in explaining the surgical success rate and is in coherence with outcomes with other studies [6-8].

In a retrospective case analysis of 37 eyes with PPC, Chan., et al. [7] observed a sensitivity of $87.5 \%$ and specificity of $62.1 \%$ and Pavan., et al. [6] observed a sensitivity 100\% and the specificity was $94.9 \%$ in identifying preexisting tears. Our results are in comparison with Pavan., et al. [6] and Sachdev., et al. [8] for the Optopol ASOCT with a sensitivity $96.77 \%$ and a specificity $90 \%$ each, While the Ziess ASOCT is in comparison with Chan., et al. (sensitivity: 90.32\% and specificity: 66.27\%). Both the ASOCT machine protocols offer a high predictive value in identifying pre-existing PC deficit in PPC. Hence AS-OCT allowed accurate detection of intact capsules unlikely to rupture intraoperatively.

The 2 eyes which developed PCR intraoperatively occurred during Irrigation and aspiration and were managed with anterior vitrectomy and 3-piece IOL with sulcus fixation and optic capture. Sachdev., et al. highlighted that greater incidence of PCR was noted in eye with PPC size greater than $4 \mathrm{~mm}$. Similarly, Pavan G., et al. [6] observed the occurrence of PCR during irrigation and aspiration of the PPC plaque in 5 eyes in their study. It is important to note here that not all eyes with PC deficit result in a PCR, which may be hypothesized firstly due to an intact anterior vitreous hyaloid as PPC is considered a remnant of the fetal hyaloid system (tunica vasculosa lentis) [3]. With both the AS-OCT modalities we were unable to visualize the integrity of the anterior hyaloid. Secondly, a major obstruction is from the optical density of the polar plaque which may have result in an artifactual appearance of a dehiscent capsule in type 2, 3 PPC.

In our study, we highlight a levelled competence of the both the ASOCT devices for detecting PC integrity in PPC. Several advantages observed in our study with regard to the previously reported studies, include a comparable sample size, prospective design, use of two different ASOCT machine protocols to identify the PC defects in PPC. Thus, we can aptly conclude that AS-OCT provides a higher specificity and sensitivity in predicting PC deficits in PPC thus en- 
abling better surgical planning to prevent and in anticipating PCR and better management of the associated complications.

\section{Bibliography}

1. Vasavada AR and Vasavada VA. "Managing the posterior polar cataract: an update". Indian Journal of Ophthalmology 65.12 (2017): 1350.

2. Kalantan H. "Posterior polar cataract: A review". Saudi Journal of Ophthalmology 26.1 (2012): 41-49.

3. Eshaghian J and Streeten BW. "Human posterior subcapsular cataract; an ultrastructural study of the posterioly migrating cells". Archives of Ophthalmology 98 (1980): 134-143.

4. Hejtmancik JF and Datilles M. "Congenital and inherited cataracts”. In: Tasman W, Jaeger EA, editors. Duane's clinical ophthalmology. CDROM ed. Baltimore, Md.: Lippincott Williams and Wilkins (2001): 74.

5. Hayashi K., et al. "Outcomes of surgery for posterior polar cataract”. The Journal of Cataract and Refractive Surgery 29 (2003): 45-49.

6. Kumar GP., et al. "Can preoperative anterior segment optical coherence tomography predict posterior capsule rupture during phacoemulsification in patients with posterior polar cataract?". Journal of Cataract and Refractive Surgery 44.12 (2018): 1441-1445.

7. Chan TC., et al. "Application of anterior segment optical coherence tomography to identify eyes with posterior polar cataract at high risk for posterior capsule rupture". Journal of Cataract and Refractive Surgery 40.12 (2014): 2076-2081.

8. Sachdev MS., et al. "Femtosecond laser-integrated anterior segment optical coherence tomography to detect preexisting posterior capsular dehiscence and increase safety in posterior polar cataracts". Journal of Cataract and Refractive Surgery 46.2 (2020): 235-240.

\section{Assets from publication with us}

- Prompt Acknowledgement after receiving the article

- Thorough Double blinded peer review

- Rapid Publication

- Issue of Publication Certificate

- High visibility of your Published work

Website: www.actascientific.com/

Submit Article: www.actascientific.com/submission.php

Email us: editor@actascientific.com

Contact us: +919182824667 